\title{
Intractable Biliary Candidiasis in Patients with Obstructive Jaundice and Regional Malignancy: A Retrospective Case Series
}

This article was published in the following Dove Press journal:

Clinical and Experimental Gastroenterology

\author{
Abdel rahman A Al manasra $\mathbb{D}^{\prime}$ \\ Khaled Jadallah $\mathbb{D D}^{2}$ \\ Abdelwahab Aleshawi ${ }^{1}$ \\ Mamoon Al-Omari (D) $^{3}$ \\ Mwaffaq Elheis (D) $^{3}$ \\ Ahmad Reyad' \\ Jehad Fataftah $\left(\mathbb{D}^{4}\right.$ \\ Hamzeh Al-Domaidat (ID ${ }^{\prime}$ \\ 'Department of General Surgery, Faculty \\ of Medicine, Jordan University of Science \\ and Technology, Irbid, Jordan; \\ ${ }^{2}$ Department of Internal Medicine, \\ Faculty of Medicine, Jordan University of \\ Science and Technology, Irbid, Jordan; \\ ${ }^{3}$ Department of Radiology, Faculty of \\ Medicine, Jordan University of Science \\ and Technology, Irbid, Jordan; \\ ${ }^{4}$ Department of Radiology, Faculty of \\ Medicine, The Hashemite University, \\ Zarqa, Jordan
}

Background: Candida species are infrequently grown in bile cultures. An association between biliary candidiasis and regional malignancy may exist. The role of fungus membranes in frequent biliary stent occlusion is also presented in this case series.

Methods: We retrospectively identified patients who underwent percutaneous trans-hepatic cholangiogram (PTC) for obstructive jaundice between January 2014 and January 2019. The results of bile cultures - obtained by PTC - for all patients were analyzed, and patients with fungus growth were determined; their medical records were reviewed.

Results: A total of 71 patients with obstructive jaundice underwent PTC between January 2015 and January 2019. Five patients (all male; mean age 55.8 years) had candida species growth in bile cultures. Two patients were diagnosed with cholangiocarcinoma, one with adenocarcinoma of the head of the pancreas, one with gallbladder cancer, and one with locally advanced gastric adenocarcinoma. Formation of fungal balls predisposed to frequent PTC drain clogging. Eradication of Candida was achieved in 4 patients after 10 days to 3 weeks of antifungal therapy.

Conclusion: We present a case series of biliary candidiasis in patients with obstructive jaundice and regional malignancy. We suggest that patients with obstructive jaundice and regional malignancy should be screened for biliary candidiasis. Persistent cholestasis may be caused by the recurrent formation of fungal membranes (balls).

Keywords: Candida, obstructive jaundice, cholangiocarcinoma, biliary, PTC

\section{Introduction}

Fungi represent an important cause of infection to human being. Candida albicans is the most pathogenic yeast species. ${ }^{1}$ Candida parapsilosis, Candida glabrata, Candida tropicalis and the newly identified (1995) species of Candida dubliniensis (Dublin, Ireland $)^{2}$ have also been associated with most forms of candidiasis. Fungal infections are frequently recognized, especially in patients with risk factors including cystic fibrosis, long term antibiotic therapy, prolonged critical care admission, and immunosuppression. ${ }^{3}$ Fungal involvement of the biliary tract seems to occur more often nowadays, probably due to the greater use of biliary interventions, both endoscopic and percutaneous. ${ }^{4}$ Data concerning the microbiological flora of the biliary tract are scarce, probably due to methodological limitations. ${ }^{4}$ Although bile analysis is useful to guide the therapeutic procedures in patients with biliary infections, few studies performed a microbiological analysis of bile in patients with different biliary diseases (Figure 1).
Correspondence: Abdel rahman A Al manasra

Department of General Surgery, Faculty of Medicine, Jordan University of Science and Technology, P.O. Box 3030, Irbid, Jordan

Tel +962 796453510

Email abdjust@yahoo.com 


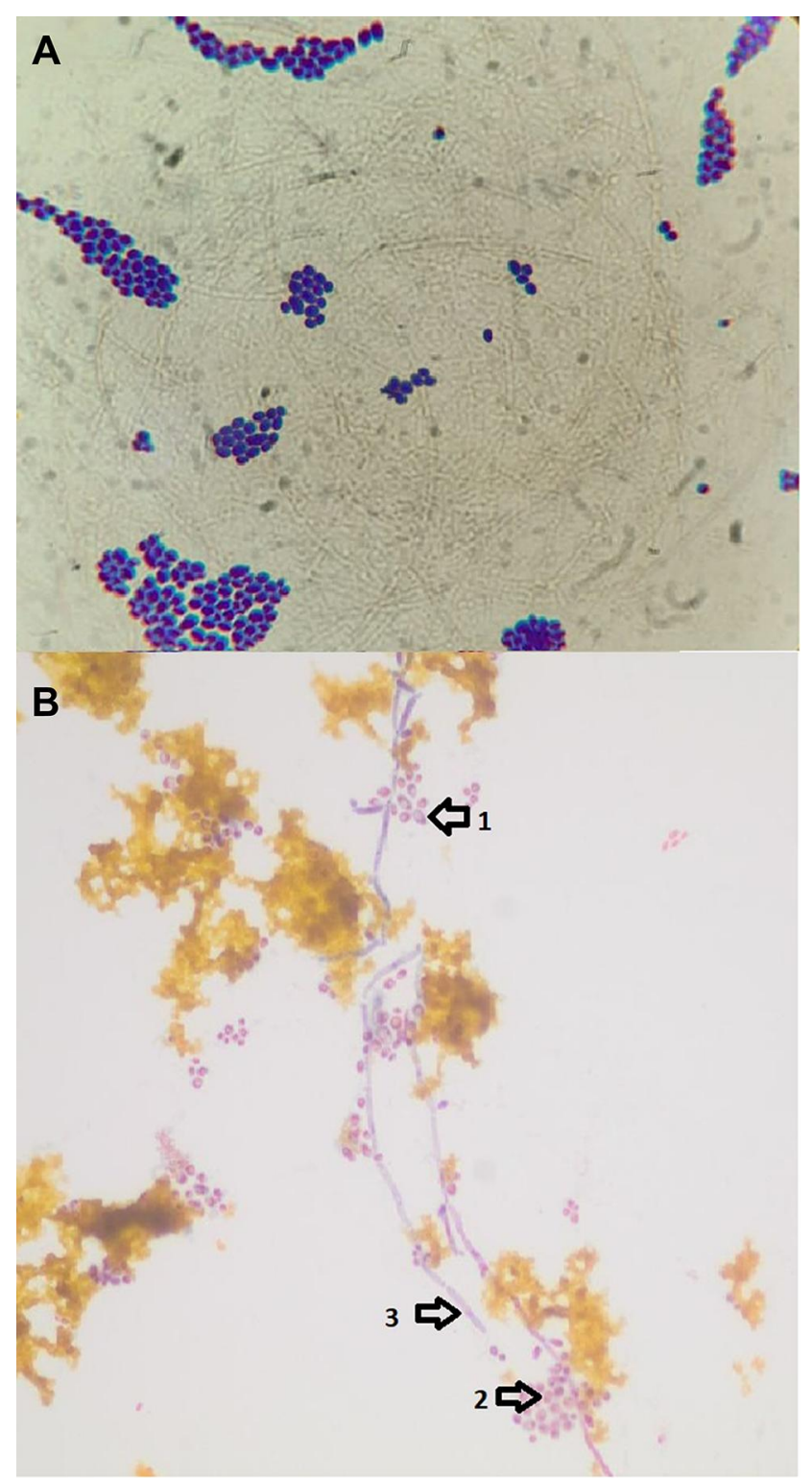

Figure I Light microscopy for histopathology smears of infected bile (400x), (A) budding yeast (B) Candida albicans demonstrating chlamydospores (arrow I), blastospores (arrow 2) and pseudohyphae (arrow 3).

Despite the emerging awareness of biliary candidiasis, its clinical relevance and potential risk factors remain debatable The terms "Biliary candidiasis" or "Biliary mycosis" are alternatively used when candida species is detected in microbiological bile fluid analysis, with or without cholangitis. However, it is still controversial whether positive bile analysis represents fungal infection or colonization, and how the results should impact the therapeutic approach. In this study, we review cases of biliary candidiasis in the setting of biliary or regional malignancy, highlighting the potential correlation between biliary candidiasis and malignancy. Additionally, we describe the role of fungus balls in frequent biliary stent occlusion.

\section{Patients and Methods}

\section{Study Design}

This is a retrospective study in which we identified patients who underwent percutaneous trans-hepatic cholangiogram (PTC) for obstructive jaundice between January 2015 and January 2019. The results of bile cultures for all patients were examined.

\section{Inclusion Criteria}

Only patients with fungus growth in bile cultures were included. The medical records of these patients were reviewed, and definitive diagnosis, management protocols, and outcomes were described.

\section{Ethics}

The study protocol was reviewed and approved by the Institutional Review Board at King Abdullah University Hospital and by the Committee of Research on Human Subjects at the Jordan University of Science and Technology. (Non-funded research No: 20200438). We protected Patients' confidentiality in accordance with declaration of Helsinki provisions. Written informed consent was obtained from the patients for the inclusion of their clinical data within this work.

\section{Procedures}

Endoscopic retrograde cholangiopancreatography (ERCP), as the intervention of choice, was attempted by an experienced gastroenterologist in those 5 patients. Inability to achieve biliary access in those patients through ERCP was attributed to the high-grade blockage of the distal portion of the common bile duct. ${ }^{5}$

Therefore, PTC was performed next, in which the level of obstruction was demonstrated, bypassed, and balloondilated. Bile samples for cultures for bacteria and fungi were obtained, and biopsies and/or brush cytology were taken. Occasionally, Candida pseudohyphae were directly observed on bile smears. We submitted samples for fungal culture in patients with a high-grade obstruction or prolonged jaundice. The specimens were cultivated both on Kimmig agar plates and in Sabouraud bouillons and additional delineation was performed by micromorphological and biochemical methods.

During initial PTC, internal plastic stents were placed without the routine use of external drainage, given that distal contrast runoff was smooth and free. After stent placement, an initial drop in cholestatic indices (direct 


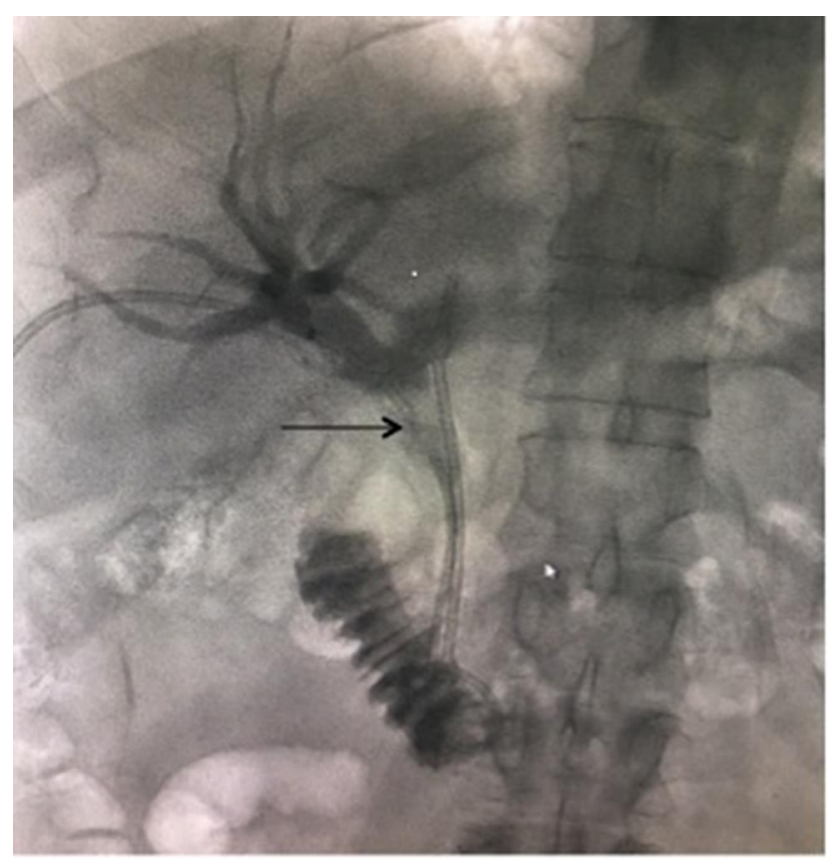

Figure 2 Common bile duct stent partial occlusion with filling defects (fungus balls) at the tip of the black arrow.

bilirubin, alkaline phosphatase, gamma-GT) level was observed in nearly all patients, but then it spiked again in 48 to $72 \mathrm{hrs}$. A repeat PTC confirmed partial or complete blockage of the stent with filling defects (fungus balls) in the common bile duct (Figure 2). Therefore, bile samples were resubmitted and an internal-external biliary drain was left in place. The daily drainage through the external catheter ranged from one to two liters, indicating persistent or recurrent occlusion. Multiple PTC stent revisions were necessary for several patients as shown in Table 1.

Following the diagnosis of fungal cholangitis with direct visualization of pseudohyphae in smears or positive culture growth, we started systemic antifungals with fluconazole $200 \mathrm{mg} /$ day and twice-daily drain flush with $100 \mathrm{mg}$ of fluconazole until repeat culture showed no growth.

\section{Results}

A total of 71 patients (mean age: 55.8, M: F 5:0) with obstructive jaundice underwent PTC between January 2015 and January 2019. Only 5 patients (7\%) had candida growth on PTC-obtained bile cultures (4 Candida albicans, 1 Candida glabrata). Two patients were diagnosed with cholangiocarcinoma, one with adenocarcinoma of the head of the pancreas, one with gallbladder cancer, and one with gastric adenocarcinoma. The patient with pancreatic adenocarcinoma experienced fungal cholangitis, which was successfully eradicated, and then he underwent a pancreaticoduodenectomy (Whipple's procedure). The rest of the patients had metastatic disease at the time of diagnosis and were referred -after fungus eradication- for chemotherapy. Table 2 summarizes the clinical and demographic features of our cases. Patients' comorbidities, disease staging, treatment options, and outcomes are illustrated in Table 1. Formation of fungal membranes predisposed to frequent drain clogging. Eradication was achieved in 4 patients after 10 days, 2 months, 2 weeks, and 3 weeks. One patient died after 6 months with persistent candida growth and frequent stent occlusion. Concerning the other 4 patients, chemotherapy delay intervals correlated with the aforementioned eradication periods.

\section{Discussion}

In this series, although we cultured candida species in $7 \%$ of patients undergoing PTC, we demonstrated that all patients with biliary candidiasis are found to have biliary or regional malignancy. In spite of the fact that other predisposing factors -including immunodeficiency, biliary instrumentation, and prolonged stagnation due to stricturecontribute to fungus overgrowth inside bile ducts, this rare infection was alarming for the presence of cancer.

Fungal cholangitis, as a form of Invasive candidiasis, is a leading cause of sepsis (candidemia) in this population, ie; patients with locoregional malignancies. Given the high cost of antifungal drugs, the potential for toxicity with prolonged use, and the poorer outcomes associated with delayed therapy; it is highly recommended that clinical microbiology as well as infectious diseases services are incorporated in the diagnosis and management of invasive candidiasis. The guidelines published by National Institute for Health and Care Excellence (NICE) ${ }^{6}$ along with 2019 updates on the core elements of hospital antibiotic Stewardship programs, both emphasized the importance of tracking, monitoring, and reporting of antimicrobial impact and outcomes. ${ }^{7}$

Welch has first described microbial presence in bile more than 130 years ago when Bacteria implication in gallstone pathogenesis was revealed. ${ }^{8,9}$ Pathogens may enter the biliary tree by a retrograde ascent from the duodenum -where 100-1000 bacteria live in each 1 milliliter of its' lining mucosa- or from portal venous blood or during instrumentation of $\mathrm{CBD} .^{10}$

Because of the existence of biliary sphincter, bile flow, and some bile components that have antibacterial features, 


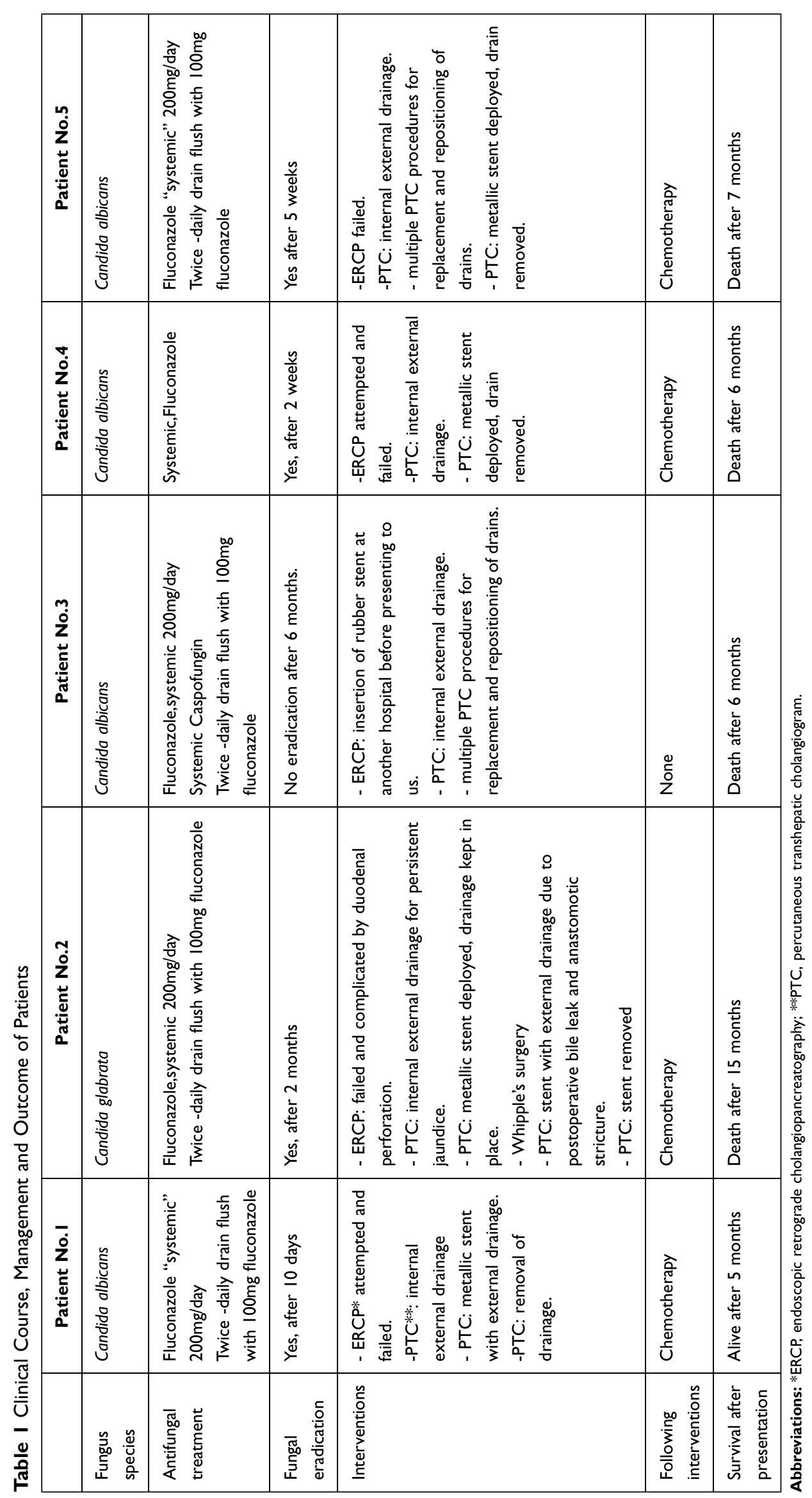


Table 2 Clinical and Demographic Features of Patients

\begin{tabular}{|l|c|c|c|c|c|c|}
\hline $\begin{array}{l}\text { Patient } \\
\text { No. }\end{array}$ & Age & Gender & $\begin{array}{c}\text { Clinical } \\
\text { Presentation }\end{array}$ & Primary Diagnosis & Disease Stage (Radiologic) & Comorbidities \\
\hline 1 & 71 & Male & $\begin{array}{c}\text { Obstructive } \\
\text { jaundice }\end{array}$ & Gallbladder Cancer & $\begin{array}{c}\text { Extensive distant lymph node } \\
\text { involvement and mesenteric } \\
\text { deposits }\end{array}$ & $\begin{array}{c}\text { Previous History of } \\
\text { urinary bladder Cancer }\end{array}$ \\
\hline 2 & 51 & Male & $\begin{array}{c}\text { Obstructive } \\
\text { jaundice }\end{array}$ & $\begin{array}{c}\text { Adenocarcinoma of the } \\
\text { head of the pancreas }\end{array}$ & No evidence of metastasis Stage Ib & $\begin{array}{c}\text { Smoker, FAP mutation } \\
\text { with history of colectomy }\end{array}$ \\
\hline 3 & 54 & Male & $\begin{array}{c}\text { Obstructive } \\
\text { jaundice, } \\
\text { abdominal pain }\end{array}$ & $\begin{array}{c}\text { Distal } \\
\text { cholangiocarcinoma }\end{array}$ & Pleural and lungs metastasis & Diabetes Mellitus \\
\hline 4 & 40 & Male & $\begin{array}{c}\text { Obstructive } \\
\text { jaundice, epigastric } \\
\text { pain }\end{array}$ & $\begin{array}{c}\text { Advanced gastric } \\
\text { cancer }\end{array}$ & Liver metastasis and extensive \\
Lymph node involvement & Smoker \\
\hline 5 & 63 & Male & $\begin{array}{c}\text { Obstructive } \\
\text { jaundice, epigastric } \\
\text { pain }\end{array}$ & $\begin{array}{c}\text { Adenocarcinoma of the } \\
\text { head of the pancreas }\end{array}$ & Multiple liver metastatic lesions & Hypertension \\
\hline
\end{tabular}

Abbreviation: FAP, familial adenomatous polyposis.

such as bile acids and immunoglobulin A ( $\operatorname{IgA}$ ), many authors suggest that bile in the biliary tract would be normally sterile unless the bile ducts are obstructed, or stones are present. ${ }^{11}$ On the contrary, multiple bacterial strains that can grow in bile (bile-tolerant) have been recently identified with the application of next-generation sequencing. ${ }^{12}$ These strains may induce chronic infection with possible malignant transformation (eg Helicobacter species, Hemophilus influenzae, E. coli). ${ }^{13}$

Moreover, in a study of 19 bile samples obtained during surgery from patients with a normal biliary tree (no stones or obstruction), there was positive growth in 16/19 samples for bacteria which led to a conclusion that human bile is not, as first thought, sterile. ${ }^{14}$

Most studies were conducted on abnormal biliary systems or unhealthy individuals. To make more reliable conclusions on the normal flora of the biliary system we believe that further studies of the normal biliary system of healthy individuals are warranted. However, this type of study may face technical challenges or ethical barriers. Therefore, we recommend obtaining bile cultures in all cases of biliary obstruction, and we believe that fungus growth in bile cultures obtained by PTC is of clinical significance.

In patients with obstructive jaundice, ERCP is usually attempted first. According to the American Society for Gastrointestinal Endoscopy (ASGE) guidelines, ${ }^{15}$ ERCP is the intervention of choice due to its advantage of drainage without the need for an external catheter. However, ERCP has a failure rate of $10-15 \% .{ }^{16}$ Common reported causes of ERCP failure include duodenal diverticula, which increases with age, adhesions due to previous upper abdominal surgery or gastrointestinal diversions, or to a less extent obstruction due to tumors. ${ }^{17}$ The failure rate increases by 78 -fold in patients with periampullary tumors that infiltrate the ampulla of Vater. Hence, PTC is a suitable alternative, especially in patients with a hilar obstruction or surgically-altered anatomy. ${ }^{18}$

Since bile samples from PTC are less prone to contamination by intestinal flora than ERCP samples, cultures from PTC samples are more reliable for an accurate diagnosis and antimicrobial selection. ${ }^{19}$

Several studies ${ }^{10,20,21}$ have investigated biliary candidiasis in terms of clinical presentation, diagnosis, and treatment modalities. However, only a few studies have analyzed its correlation with malignancy ${ }^{22}$ and the impact on patient outcomes, including survival rates. In a study by Kim and colleagues, ${ }^{18}$ Candida species were found in $23 \%$ of bile samples collected during PTC from patients with cholangiocarcinoma; Patients with biliary candidiasis had reduced survival compared to those without, the authors suggested that biliary candidiasis is a factor of poor prognosis. Reduced survival is probably attributed to factors such as sepsis, poor oral intake, complications of frequent invasive interventions, and delayed surgery or chemotherapy. 
In another study by Lenz and colleagues, ${ }^{4}$ in which bile samples were collected during ERCP, Candida was isolated in $30 \%$ of patients. However, all patients suffered from severe chronic diseases and required frequent intervention; the authors found in a multivariate analysis that previous endoscopic sphincterotomy -which eliminates an important protective mechanism- was a significant risk factor for biliary candidiasis.

Other studies have demonstrated that fungus balls could be responsible for frequent CBD and stent occlusion. Story and Gluck $^{23}$ described the soft filling defects within the bile duct. They also outlined the treatment approach, which consisted of daily oral antifungal and daily antifungal flushing through PTC external drain or cholecystostomy tube. In that study, the proposed treatment succeeded in the eradication of the infection in about $75 \%$ of cases.

In the present study, the diagnosis of fungal cholangitis preceded that of malignancy, suggesting that biliary candidiasis could be a warning sign of an underlying malignancy. We also highlight the role of fungus balls in frequent stent occlusion after PTC or ERCP, which is a rare but potentially treatable condition. Additionally, we emphasize the role of combined (systemic and intrabiliary) antifungal therapy in eradicating the pathogen. Our results suggest that biliary candidiasis could be associated with a worse prognosis.

This study has some limitations. Firstly, it is a retrospective study. Secondly, the sample size of patients with biliary candidiasis was too small to evaluate risk factors with statistical significance. Thirdly, many patients who did not have their bile cultures obtained may still have the infection, leading to some false-negative cases. Despite these limitations, the findings in this study contribute to the understanding of cancer association with biliary candidiasis and the importance of the implementation of an appropriate treatment approach.

In Conclusion, sampling bile for culture is recommended in patients with obstructive jaundice. Biliary candidiasis should raise concerns of underlying biliary or regional malignancy, in particular in the absence of other risk factors. Persistent cholestasis after biliary drainage may be attributed to fungus growth in the form of fungal balls, which may delay treatment by chemotherapy or surgery. Future prospective studies are warranted to investigate the putative association between biliary candidiasis and malignancy.

\section{Disclosure}

The authors report no conflicts of interest for this work.

\section{References}

1. Moran GP, Coleman DC, Sullivan DJ. Candida albicans versus Candida dubliniensis: why is Calbicans more pathogenic? Int J Microbiol. 2012;2012:205921. doi:10.1155/2012/205921

2. Krcmery V, Barnes AJ. Non-albicans Candida spp. causing fungaemia: pathogenicity and antifungal resistance. $J$ Hosp Infect. 2002;50 (4):243-260. doi:10.1053/jhin.2001.1151

3. Lenz P, Eckelskemper F, Erichsen T, et al. Prospective observational multicenter study to define a diagnostic algorithm for biliary candidiasis. World J Gastroenterol. 2014;20(34):12260-12268. doi:10.3748/wjg.v20.i34.12260

4. Lenz P, Conrad B, Kucharzik T, et al. Prevalence, associations, and trends of biliary-tract candidiasis: a prospective observational study. Gastrointest Endosc. 2009;70(3):480-487. doi:10.1016/j. gie.2009.01.038

5. Swan MP, Bourke MJ, Williams SJ, et al. Failed biliary cannulation: clinical and technical outcomes after tertiary referral endoscopic retrograde cholangiopancreatography. World $J$ Gastroenterol. 2011;17(45):4993-4998. doi:10.3748/wjg.v17.i45.4993

6. National Institute for Health and Care Excellence. Guideline NG15. Antimicrobial stewardship: systems and processes for effective antimicrobial medicine use. Available from: https://www.nice.org.uk/gui dance/ng15/resources/antimicrobial-stewardship-systemsandprocesses-for-effective-antimicrobial-medicine-use-1837273110469. Accessed 8, 2021.

7. CDC. Core elements of hospital antibiotic Stewardship programs. Atlanta, GA: US Department of Health and Human Services, CDC; 2019. Available from: https://www.cdc.gov/antibiotic-use/coreelements/hospital.html. Accessed February 8, 2021.

8. Keighley MR. Micro-organisms in the bile. A preventable cause of sepsis after biliary surgery. Ann $R$ Coll Surg Engl. 1977;59 (4):328-334.

9. Wetter LA, Hamadeh RM, Griffis JM, et al. Differences in outer membrane characteristics between gallstone-associated bacteria and normal bacterial flora. Lancet. 1994;343(8895):444-448. doi:10.1016/S0140-6736(94)92691-3

10. Shenoy SM, Shenoy S, Gopal S, Tantry BV, Baliga S, Jain A. Clinicomicrobiological analysis of patients with cholangitis. Indian J Med Microbiol. 2014;32:157-160. doi:10.4103/0255-0857.129802

11. Suna N, Yildız H, Yüksel M, et al. The change in microorganisms reproducing in bile and blood culture and antibiotic susceptibility over the years. Turk $J$ Gastroenterol. 2014;25:284-290. doi:10.5152/tjg.2014.6253

12. Ye F, Shen H, Li Z, et al. Influence of the biliary system on biliary bacteria revealed by bacterial communities of the human biliary and upper digestive tracts. PLoS One. 2016;11(3):e0150519. doi:10.1371/ journal.pone. 0150519

13. Ljungh $\AA$, Wadström T. The role of microorganisms in biliary tract disease. Curr Gastroenterol Rep. 2002;4:167-171. doi:10.1007/ s11894-002-0055-6

14. Warburton RC, Gelson W, Harper S, et al. Human bile retrieved from the normal biliary system is not sterile. J Hepatol. 2017;1(66):S63S94.

15. Buxbaum JL, Abbas Fehmi SM, Sultan S, Fishman DS, Qumseya BJ, Cortessis VK. ASGE guideline on the role of endoscopy in the evaluation and management of choledocholithiasis. Gastrointest Endosc. 2019;89(6):1075-1105.e15. doi:10.1016/j.gie.2018.10.001

16. Collins C, Maguire D, Ireland A, Fitzgerald E, O'Sullivan GC. A prospective study of common bile duct calculi in patients undergoing laparoscopic cholecystectomy. Ann Surg. 2014;239:28-33. doi:10.1097/01.sla.0000103069.00170.9c

17. Balik E, Eren T, Keskin M, et al. Parameters that may be used for predicting failure during endoscopic retrograde cholangiopancreatography. J Oncol. 2013;2013:201681. doi:10.1155/ 2013/201681 
18. Kim IH, Choi JK, Lee DG, et al. Clinical significance of isolated biliary candidiasis in patients with unresectable cholangiocarcinoma. Hepatobiliary Pancreat Dis Int. 2016;15:533-539. doi:10.1016/ S1499-3872(16)60109-1

19. Negm AA, Schott A, Vonberg RP, et al. Routine bile collection for microbiological analysis during cholangiography and its impact on the management of cholangitis. Gastrointest Endosc. 2010;72 (2):284-291. doi:10.1016/j.gie.2010.02.043

20. Neve RA, Biswas S, Dhir V, Mohandas KM, Kelkar R, Shukla P. Bile cultures and sensitivity patterns in malignant obstructive jaundice. Indian J Gastroenterol. 2003;22(1):16-18.
21. Diebel LN, Raafat AM, Dulchavsky SA, Brown WJ. Gallbladder and biliary tract candidiasis. Surgery. 1996;120(4):760-764. doi:10.1016/ S0039-6060(96)80028-6

22. Yu H, Guo Z, Xing W, Guo X, Liu F, Li B. Bile culture and susceptibility testing of malignant biliary obstruction via PTBD. Cardiovasc Intervent Radiol. 2012;35:1136-1144. doi:10.1007/ s00270-011-0263-2

23. Story B, Gluck M. Obstructing fungal cholangitis complicating metal biliary stent placement in pancreatic cancer. World J Gastroenterol. 2010;16(24):3083-3086. doi:10.3748/wjg.v16.i24.3083

\section{Publish your work in this journal}

Clinical and Experimental Gastroenterology is an international, peerreviewed, open access, online journal publishing original research, reports, editorials, reviews and commentaries on all aspects of gastroenterology in the clinic and laboratory. This journal is indexed on American Chemical Society's Chemical Abstracts Service (CAS)
The manuscript management system is completely online and includes a very quick and fair peer-review system, which is all easy to use. Visit http://www.dovepress.com/testimonials.php to read real quotes from published authors.

Submit your manuscript here: https://www.dovepress.com/clinical-and-experimental-gastroenterology-journal 